\title{
Pole-Zero Matching Technique for Multilayered Periodic Structures with Application to the Design of Reflectarray Antennas
}

R. Florencio
R. R. Boix

\author{
J. A. Encinar
}

\begin{abstract}
In order to design reflectarray antennas within reasonable CPU times, fast and accurate numerical tools for the analysis of periodic multilayered structures are required. In this paper, a pole-zero matching technique is applied to the determination of the scattering matrix of periodic arrays of stacked rectangular microstrip patches as a function of the dimensions of the patches. The pole-zero matching technique makes it possible to obtain closed-form equations for the scattering matrix of the periodic structures from a reduced set of full-wave data. These closed-form equations are very useful because they lead to important CPU time savings when they are applied to the design of reflectarray antennas. In fact, in this paper we present a comparison between a reflectarray design based on a fullwave analysis of each element and a reflectarray design based on the derived closed-form equations. It is shown that the CPU time required by the full-wave approach is nearly one order of magnitude larger than that required by the approach based on closed-form equations ${ }^{1}$.
\end{abstract}

Index Terms-Reflectarray antennas, multilayered reflectarrays, periodic structures, pole-zero matching.

\section{INTRODUCTION}

A printed reflectarray is a planar reflector made of one or more layers of microstrip patch arrays. The microstrip patches are conveniently tuned to produce a progressive phase shift of the reflected field, and this makes it possible to collimate or to conform the radiated beam when the reflectarray is illuminated by a feed (usually a horn antenna), just as it happens with conventional reflectors [1]. Printed reflectarrays present several technological advantages when comparing with conventional reflectors and phased arrays, such as low cost (specially in the case of contoured beam designs), elimination of complex feed networks, possibility of use in dual polarization applications with different beam shaping in each polarization, and possibility of use in steerable or reconfigurable beam applications at a moderate cost (by insertion of controllable devices such as PIN diodes, MEMs switches, varactors, etc).
One of the possible implementations to obtain a progressive phase shift of the reflected field consists of varying the resonant length of the microstrip patches [2]-[5]. This technique presents the advantage of an easy manufacturing based on conventional photo-etching process. In fact, the technique can be used for large reflectarrays at microwave and millimeter-wave frequencies, and it leads to lower crosspolarization levels than those achieved with stubs of different length attached to the radiating patches [1].

A crucial stage in the design of reflectarray antennas made of patches of variable size is the choice of the sizes of the patches that lead to the appropriate reflection phases for the generation of a certain radiation pattern. When choosing the size of every patch, it is customary to assume that each set of stacked patches is surrounded by an infinite periodic array of stacked patches of the same size. This is known as the local periodicity assumption [1], and it makes it possible to design reflectarray antennas within reasonable CPU times. The validity of the local periodicity assumption is based on the fact that it leads to numerical results that show good agreement with experimental results [3]-[7]. In the design of reflectarray antennas under the local periodicity assumption, the electromagnetic analysis of the scattering of a plane wave by periodic multilayered structures is typically performed tens of thousands of times, or even more if an optimization is run to enforce the phase requirements at different frequencies as in [6]-[7]. Therefore, to avoid the consumption of prohibitive CPU times, it is necessary to have efficient tools for numerical analysis of such periodic structures.

Despite the extensive literature available on the efficient tools for the analysis of periodic multilayered structures, there is relatively limited published material on the inverse problem of the synthesis which aims to derive the size of patches required to produce a certain phase shift in the reflection coefficient of those periodic structures. In order to improve the synthesis procedure, in this work we propose a polezero matching technique which makes it possible to obtain 
closed-form expressions for the scattering matrix of periodic arrays of stacked rectangular microstrip patches in terms of the dimensions of the patches. A comparison is carried out between the CPU time required for the design of a two-layer reflectarray with the procedure reported in [5] and the CPU time required by the closed-form equations derived in this paper. The results obtained show that the use of the mentioned closed-form equations leads to important CPU time savings.

\section{POLE-ZERo MATCHING TECHNIQUE}

The authors of [8] present a pole-zero matching technique to obtain a frequency dependent rational approximation of the admittance matrix of a doubly periodic infinite array of dipoles from a reduced number of full wave simulations. The justification for this rational approximation is provided by Foster's theorem. The same concept is used in [9] to obtain a similar rational approximation of the admittance matrix of the array of dipoles as a function of the dipole length. In this section we apply the same principles to the determination of a rational approximation of the scattering matrix of a periodic array of stacked rectangular microstrip patches as a function of the patch dimensions.

Figs. 1(a) and (b) show a periodic structure consisting of two arrays of stacked rectangular patches embedded in a multilayered substrate. Each layer has a thickness $h_{i}(i=1, \ldots, N C)$ and complex permittivity $\varepsilon_{i}=\varepsilon_{0} \varepsilon_{r i}\left(1-j \tan \delta_{i}\right)$. The periodic structure is illuminated by a plane wave. To avoid grating lobes, the dimensions of the unit cell, $a$ and $b$, are chosen in such a way that the restrictions $a<\lambda_{0} /\left(1+\operatorname{sen} \theta_{\text {inc }}\right)$ and $b<\lambda_{0} /\left(1+\operatorname{sen} \theta_{\text {inc }}\right)$ are fulfilled, where $\lambda_{0}$ is the free space wavelength. These restrictions on the unit cell dimensions ensure that when the reflected electric field is expanded in terms of Floquet space harmonics with wavenumber vector components $k_{x m}=k_{0} \sin \theta_{\text {inc }} \cos \phi_{\text {inc }}+2 \pi m / a\left(k_{0}=2 \pi / \lambda_{0}\right)$ and $k_{y n}=k_{0} \sin \theta_{\text {inc }} \cos \phi_{\text {inc }}+2 \pi n / b$, the space harmonic $m=0, n=0$ will be the only propagating wave for any incident angle $\theta_{\text {inc }}$ and the rest of the space harmonics will attenuate. Under these conditions, we can describe the incident and reflected total propagating electric field using two z-directed transmission lines with propagation constant $k_{z}=\sqrt{k_{0}^{2}-\left(k_{0} \sin \theta_{\text {inc }}\right)^{2}}$ and characteristic admittances $Y_{0, \mathrm{TE}}=\frac{k_{z}}{\omega \mu_{0}}$ and $Y_{0, \mathrm{TM}}=\frac{\omega \epsilon_{0}}{k_{z}}$ for TE and TM polarizations respectively [8].

As shown in [8], in the general case where the incidence direction is out of the principal planes $\left(\phi_{\text {inc }} \neq n \cdot 90^{\circ}, n=\right.$ $0,1,2,3)$, the response of the structure can be modeled as a two-port network where each port represents one of the two polarizations TE and TM [see Fig. 1(c)]. This makes it possible to account for the coupling between the two polarizations. The scattering matrix of the two-port network, $\overline{\overline{\mathbf{S}}}_{\mathrm{TE}, \mathrm{TM}}$, can be defined by means of the expression

$$
\left(\begin{array}{l}
E_{0, \mathrm{TE}}^{\mathrm{ref}} \\
E_{0, \mathrm{TM}}^{\mathrm{ref}}
\end{array}\right)=\overline{\overline{\mathbf{S}}}_{\mathrm{TE}, \mathrm{TM}} \cdot\left(\begin{array}{c}
E_{0, \mathrm{TE}}^{\mathrm{inc}} \\
E_{0, \mathrm{TM}}^{\mathrm{inc}}
\end{array}\right)
$$

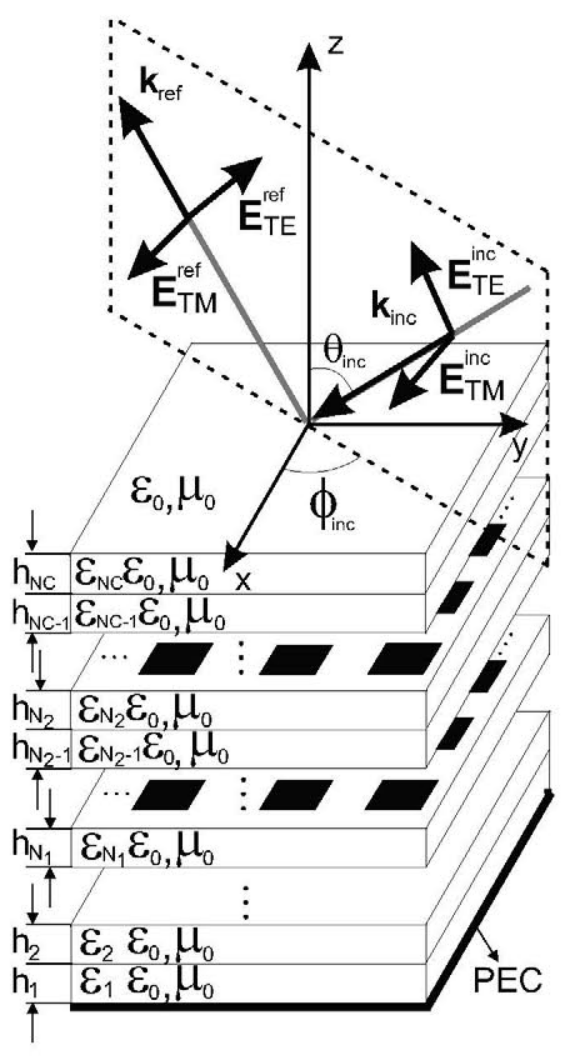

(a)

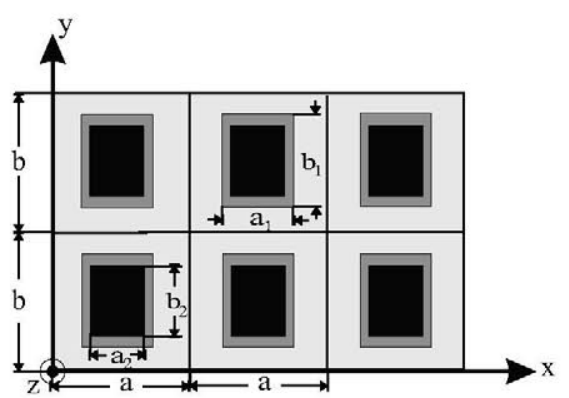

(b)

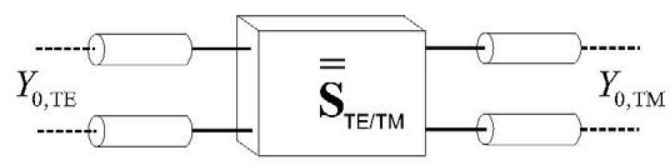

(c)

Fig. 1. (a) Plane wave impinging on a periodic structure with two arrays of stacked patches embedded in a multilayered substrate. (b) Top view of the periodic structure. (c) Two-port network model of the periodic structure. 
where

$$
\overline{\overline{\mathbf{S}}}_{\mathrm{TE}, \mathrm{TM}}=\left(\begin{array}{ll}
S_{\mathrm{TE}, \mathrm{TE}} & S_{\mathrm{TE}, \mathrm{TM}} \\
S_{\mathrm{TM}, \mathrm{TE}} & S_{\mathrm{TM}, \mathrm{TM}}
\end{array}\right)
$$

Following [8] and [9], for fixed values of the ratios $a_{1} / b_{1}$, $a_{2} / b_{2}$ and $a_{2} / a_{1}, a, b$, frequency and incidence angle, we propose that the elements of the scattering matrix $\overline{\overline{\mathbf{S}}}_{\mathrm{TE}, \mathrm{TM}}$ can be expressed as rational functions of the patch dimension $a_{1}$ as shown below

$$
\begin{aligned}
& S_{i j}\left(a_{1}\right)=\frac{C_{0, i j}+C_{1, i j}\left(a_{1}\right)+\ldots+C_{N, i j}\left(a_{1}\right)^{N}}{1+D_{1, i j}\left(a_{1}\right)+\ldots+D_{M, i j}\left(a_{1}\right)^{M}} \\
& i, j=\text { TE,TM }
\end{aligned}
$$

where the coefficients $C_{0, i j}, \ldots, C_{N, i j}, D_{1, i j}, \ldots, D_{M, i j}$ will be in general complex numbers.

Whereas in [8] the coefficients $C_{r, i j}(r=0, \ldots, N)$ and $D_{s, i j}(s=1, \ldots, N)$ are obtained by identification of the poles and zeros of $S_{i j}\left(a_{1}\right)$ as a function of $a_{1}$, in this paper those coefficients have been obtained by means of a least square scheme with singular value decomposition [10]. This has made it possible to achieve an accurate determination of the coefficients from a small number of full-wave analysis of the periodic multilayered structure. For the full-wave analysis of the periodic structure, we have employed the spectral domain method of moments hybridized with a generalized scattering matrix approach [11].

In order to design reflectarray antennas under the local periodicity assumption, we need to know the scattering matrix of the periodic structure in cartesian coordinates [1]. Since the scattering matrix of (2) is expressed in terms in the TE/TM basis, we need a transformation from the TE/TM basis to the cartesian basis. This transformation is given by

$$
\overline{\overline{\mathbf{S}}}_{\mathrm{XY}}=\overline{\overline{\mathbf{P}}}_{\mathbf{E}} \cdot \overline{\overline{\mathbf{S}}}_{\mathrm{TE}, \mathrm{TM}} \cdot\left[\overline{\overline{\mathbf{P}}}_{\mathrm{E}}\right]^{-1}
$$

where

$$
\overline{\overline{\mathbf{P}}}_{\mathbf{E}}=\left[\overline{\overline{\mathbf{P}}}_{\mathrm{E}}\right]^{-1}=\left(\begin{array}{ll}
-\sin \phi_{\mathrm{inc}} & \cos \phi_{\mathrm{inc}} \\
\cos \phi_{\mathrm{inc}} & \sin \phi_{\mathrm{inc}}
\end{array}\right)
$$

\section{DESIGN OF TWO-LAYER REFLECTARRAYS}

If a horn antenna is used to feed a reflectarray and the elements of the reflectarray are assumed to be in the far-field region of the horn, the phase-shift on the $l$-th element of the reflectarray that is required to produce a reflected pencil beam in the direction $\left(\theta_{0}, \phi_{0}\right)$ is given by the expression [1]

$$
\Phi_{l}=k_{0}\left[d_{l}-\sin \theta_{0}\left(x_{l} \cos \phi_{0}+y_{l} \sin \phi_{0}\right)\right]
$$

where $d_{l}$ is the distance between the $l$-th element and the phase center of the feed, and $\left(x_{l}, y_{l}\right)$ are the coordinates of the center of the $l$-th element. In order to adjust the dimensions of the patches that provide the required phase of the reflection coefficient in each cell, we have implemented a zero finding numerical code that iteratively calls for an analysis routine.
In this paper, the analysis routine makes use of Eqns. (3) and (4) for the determination of $\overline{\overline{\mathbf{S}}}_{\mathrm{XY}}$. Since the rational approximation of (3) is obtained from a small number of full-wave analysis of the periodic structure of Fig. 1(a), the approach proposed in this paper is more convenient than that proposed in [5] where a full-wave analysis of the periodic structure is required every time the analysis routine is called. For dual polarization, the dimensions of the patches in the $x$ and $y$ directions are independently adjusted to obtain the required phase (see Eqn. (6)) for the $x$ and $y$ polarized fields. The same procedure makes it also possible the adjustment of the patch dimensions for a phase distribution different from that of Eqn. (6) with a view to obtaining conformal beams [1]. In the end, all the patch dimensions are obtained and the photo-etching masks for the two arrays are automatically generated from the output file of the design numerical code.

Let us consider the two-layer reflectarray designed and measured in [5]. This reflectarray consists of stacked rectangular patches separated by thick ROHACELL 51. Fig. 2 shows the phase of the reflection coefficient of the periodic structures used in the design of this reflectarray at $12 \mathrm{GHz}$ and normal incidence $\left(\theta_{\text {inc }}=0^{\circ}\right)$. In Fig. 2 the data obained for the phase of the reflection coefficient by means of full-wave analysis are compared with the data obtained by means of the pole-zero matching technique (Eqn. (3)) when two zeros and three poles are used in the rational function approximation $(N=2$ and $M=3$ ). Note there is a very good agreement between both sets of results, and that the relative error is around $0.1 \%$ on average.

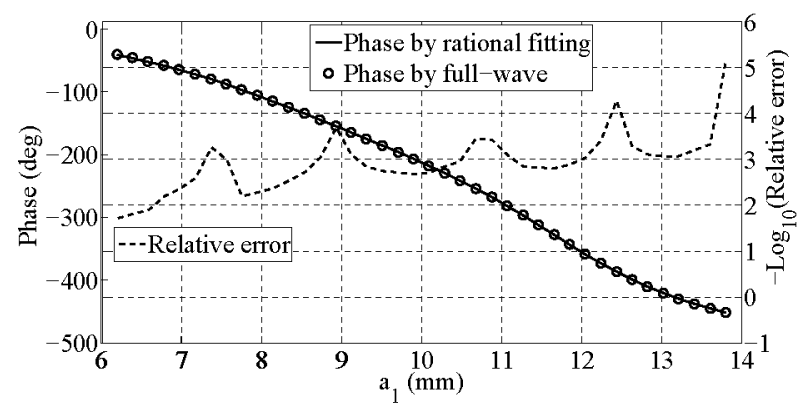

Fig. 2. Phase of reflection coefficient of the structure of Figs. 1(a) and 1(b) versus the dimensions of the patch closer to the ground plane. Parameters: $\theta_{\text {inc }}=0^{\circ} ; N C=N_{2}=2 ; N_{1}=1 ; a_{1}=b_{1} ; a_{2}=b_{2} ; a_{2}=0.7 a_{1}$ $h_{2}=h_{1}=3 \mathrm{~mm} ; \varepsilon_{r 1}=\varepsilon_{r 2}=1.05 ; \tan \delta_{1}=\tan \delta_{2}=0.001 ; N=2$; $M=3$

One two-layer circular reflectarray of $406 \mathrm{~mm}$ diameter $(29 \times 29$ elements) has been designed for dual polarization to radiate a focused beam in the direction $\theta_{0}=19^{\circ}, \phi_{0}=0^{\circ}$ at $11.95 \mathrm{GHz}$ [5]. The feed is located at coordinates $x_{f}=-116$ $\mathrm{mm}, y_{f}=0$ and $z_{f}=340 \mathrm{~mm}$. For both polarizations, the power radiation pattern of the feed is modeled as $\cos ^{q} \theta$ with $q=0.7$. The reflectarray design has been carried out by means of the conventional full-wave approach of [5], and by means of the pole-zero matching approach proposed in this work. 
The radiation patterns obtained with the two approaches have been compared in Figs. 3 and 4.

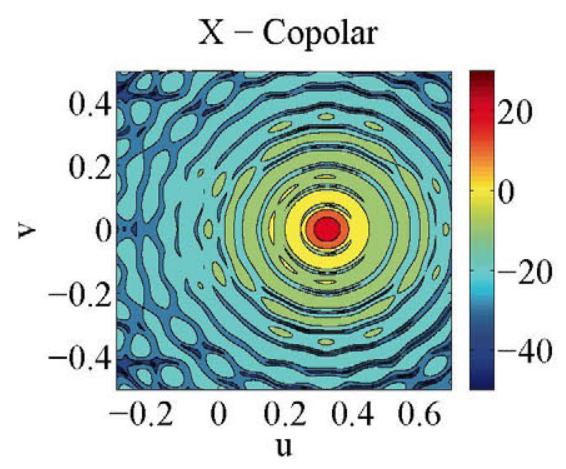

(a)

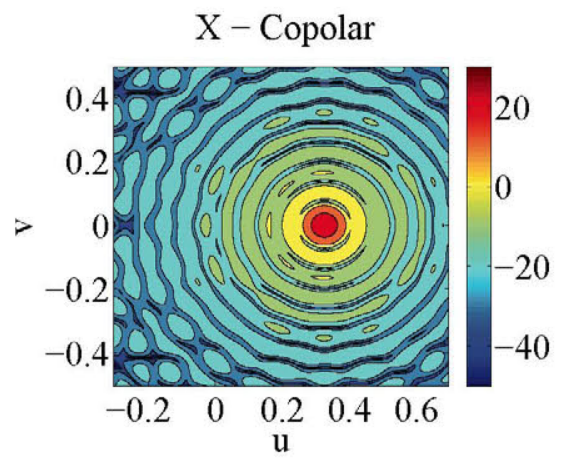

(b)

Fig. 3. Radiation pattern for the copolar component of X-polarization at $11.95 \mathrm{GHz}$. (a) Design based on the rational fitting approach. (b) Design based on the conventional full-wave technique [5].

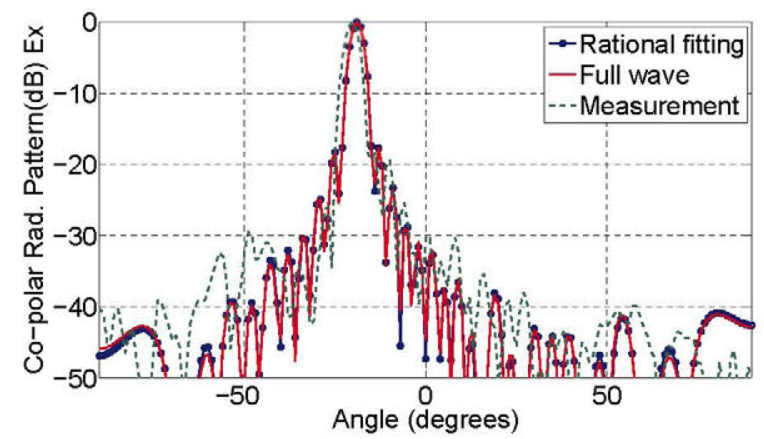

Fig. 4. Theoretical and measured radiation patterns on the $x-z$ plane for $\mathrm{X}$-polarization at $11.95 \mathrm{GHz}$.

Figs. 3 and 4 show that the results obtained for the radiation patterns of the reflectarray with the conventional full-wave approach [5] and with the rational fitting approach (Eqn. (3)) are basically the same. Also, Fig. 4 shows a good agreement is found between the numerical results and the experimental results above $-30 \mathrm{~dB}[5]$. The ratio between the CPU times required for the total design is $T_{\text {full-wave }} / T_{\text {rational fitting }}=7.8$, which indicates that the pole-zero matching technique leads to important CPU time savings.

\section{CONCLUSION}

The pole-zero matching technique proposed in [8] and [9] has been applied to the determination of the scattering matrix of periodic arrays of stacked patches in multilayered substrates as an analytical function of the patch dimensions. The only restriction in the use of this technique is that higher-order Floquet modes must be below their cutoff frequencies, but the restriction is accomplished in the case of reflectarray antennas to avoid grating lobes. The technique has been applied to the design of a two-layer reflectarray with patches of variable size under the local periodicity assumption. The results obtained for the radiation patterns of the reflectarray with the pole-zero matching technique have been compared with those obtained with the conventional full-wave approach, and good agreement has been found. Also, a comparison between the CPU times required by the two techniques reveals that the pole-zero technique leads to important CPU time savings.

\section{REFERENCES}

[1] J. Huang, J. A. Encinar, Reflectarray antennas, Piscataway, NJ/New York: IEEE Press/Wiley, 2008.

[2] D.M. Pozar, T.A. Metzler, "Analysis of a reflectarray antenna using microstrip patches of variable size", Electron. Lett., vol. 29, pp. 657658, April 1993.

[3] D. M. Pozar, S. D. Targonski, and H. D. Syrigos, "Design of milimeter wave microstrip reflectarrays," IEEE Trans. Antennas Propagat., vol. 45, pp. 287-296, Feb. 1997.

[4] D. M. Pozar, S. D. Targonski, and R. Pokuls, "A shaped-beam microstrip patch reflectarray," IEEE Trans. Antennas Propagat., vol. 47, pp. 11671173, July. 1999.

[5] J. A. Encinar, "Design of two-layer printed reflectarrays using patches of variable size," IEEE Trans. Antennas Propagat., vol. 49, pp. 1403-1410, Oct. 2001.

[6] J. A. Encinar, J. A. Zornoza, "Three-layer printed reflectarrays for contoured beam space applications", IEEE Trans. Antennas Propagat., vol. AP-52, pp. 1662-1664, May 2004.

[7] J. A. Encinar, L. Sh. Datashvili, J. A. Zornoza, M. Arrebola, M. SierraCastaer, J. L. Besada-Sanmartn, H. Baier, H. Legacy, "Dual-polarization dual-coverage reflectarray for space applications", IEEE Trans. Antennas Propagat., vol. AP-54, pp. 2827-2837, Oct. 2006.

[8] S. Maci, M. Caiazzo, A. Cucini, and M. Casaletti, "A pole-zero matching method for EBG surfaces composed of a dipole FSS printed on a grounded dielectric slab", IEEE Trans. Antennas Propagat., vol. 53, no. 1, pp. 70-81, Jan. 2005.

[9] M. Garcia Vigueras, J. L. Gomez Tornero, G. Goussetis, J. S. Gomez Diaz and A. Alvarez Melcon, "A modified pole-zero technique for the synthesis of waveguide leaky-wave antennas loaded with dipole-based FSS", IEEE Trans. Antennas Propagat., vol. 58, no. 6, pp. 1971-1979, June 2005.

[10] R. S. Adve, T. K. Sarkar, S. M. Rao, E. K. Miller, D. R. Pflug, "Application of the Cauchy method for extrapolating/interpolating narrowband system responses", IEEE Trans. Microwave Theory Tech., vol. 45, pp. 837-845, May 1997.

[11] C. Wan and J. A. Encinar, "Efficient computation of generalized scattering matrix for analyzing multilayered periodic structure," IEEE Trans. Antennas Propag., vol. 43, no. 11, pp.1233-1242, Nov. 1995. 